\title{
An Integrated Electrophoresis Microchip for Label-free Detection Using Surface Plasmon Resonance Image
}

\author{
X. Chen ${ }^{1}$, H. Y. Cai ${ }^{1}$, L. L.Zhang ${ }^{1}$, H. Li ${ }^{1}$, Y. P. Chao ${ }^{2}$, J. H. Sun ${ }^{1}$, D. F. Cui ${ }^{1}$ \\ ${ }^{1}$ State Key Laboratory of Transducer Technology, Institute of Electronics, Chinese Academy of \\ Sciences, Beijing, China \\ Corresponding author's e-mail address: chenxing2004star@yahoo.com.cn \\ ${ }^{2}$ Institute of Microbiology, Chinese Academy of Sciences, Beijing, China
}

\begin{abstract}
:
In this work, a novel microfluidic-based electrophoresis method combined with surface plasmon resonance image (SPRI) has been developed for electrokinetic fluid transport and label-free detection. The integrated microfluidic chip was designed and fabricated by MEMS technology. A PDMS layer with double T-type microchannels for electrophoresis was fabricated and then directly bonded on a specialized SPR sensing substrate. In order to avoid the electrochemical corrosion of the metallic SPR surface, a microarray of rectangle gold spots was instead of an intact gold film. In a case, FITC in TBE buffer was been electrically driven and detected in the integrated microchip using our SPRI system under $100 \mathrm{~V} / \mathrm{cm}$ electric field strength. The applied electric field with high conductivity buffers in our integrated microchip is much higher than that reported in the past literatures, which has the greatest potential for various real-time electrophoresis separation and label-free detection in the fields of genetic analysis, protein assay, environmental testing, biological warfare agent detection, drug discovery and so on.
\end{abstract}

Key words: Microfluidics; Surface Plasmon Resonance Image (SPRI), Capillary Electrophoresis (CE), Label-free Detection

\section{Introduction}

In recent years, microfluidics has become more attractive analytical methods with many advantages such as high throughput, integrated sample processing, minimized sample and reagent volume, and increased ease of fluid manipulation, which has been applied in many important fields such as clinical diagnostics, chemical analysis, food safety testing, environmental monitoring, pharmaceutical drug discovery, etc [1-5]. However these miniature chips or devices still need high sensitive and compatible detection technology.

Surface plasmon resonance (SPR) is a well established label free, real time detection technique with high sensitivity, which has been widely used for studying molecular interactions [6-7]. Surface plasmon resonance (SPR) has emerged as a powerful label-free analysis technique. In our pre-research, SPRI technology has been successfully integrated with a hard-soft microfluidic chip for biological assays [8]. The group of Tao [9] used SPR image technology combined with electrophoresis microchip for label-free protein detection and separation in real-time. However, the most of field strengths applied to the device was $30-50 \mathrm{~V} / \mathrm{cm}$, resulting in slow separation and broad peaks. Electrically driven fluid flow is problematic for standard SPR/SPR image systems mainly due to electrochemical corrosion of the SPR-sensing metal film.

In order to avoid electrochemical corrosion of the SPR-sensing substrate, the SPR-sensing gold membrane was segmented into small units and coated with a protective polymer film of CYTOP polymer with high dielectric strength [9]. With these modifications of SPR sensing substrate, the highest field strengths applied to this device was up to $50 \mathrm{~V} / \mathrm{cm}$. Moreover, it is difficult to further fix biomolecules on the surface of the SPR-sensing substrate with this protective polymer.

In this work, an integrated microchip with a specialized gold microarray was developed, in which FITC was driven by electrokinic force and measured by using our SPR image system. This new method will be widely used for electrophoresis separations with label-free detection. 


\section{Experimental}

An integrated microchip with the size of $24 \mathrm{~mm} \times 50 \mathrm{~mm}$ for electrokinetic fluid transport and SPRI detection is designed, as shown in Fig 1, which includes three layers. The top layer was made of glass with 4 holes which was used for fixing electrodes. The middle layer was made of a PDMS layer with typical double Ttype microchannels for electrophoresis process, which was fabricated by the casting method with a silicon positive mould. The sizes of the horizontal long channel were $40 \mu \mathrm{m} \times 400 \mu \mathrm{m} \times 30 \mathrm{~mm}(\mathrm{H} \times \mathrm{W} \times \mathrm{L})$ and the sizes of the two vertical short channels were $40 \mu \mathrm{m} \times 400 \mu \mathrm{m} \times 6 \mathrm{~mm}(\mathrm{H} \times \mathrm{W} \times \mathrm{L})$. The bottom layer was made of a metallic glass slide, which was used for SPR sensing. The bottom SPR sensing substrate with a rectangle gold microarray was fabricated by lift-off technology. The three layers, which were treated with oxygen plasma for increasing the sealing strength, were reversibly bonded together by using the nature characteristic of PDMS material.

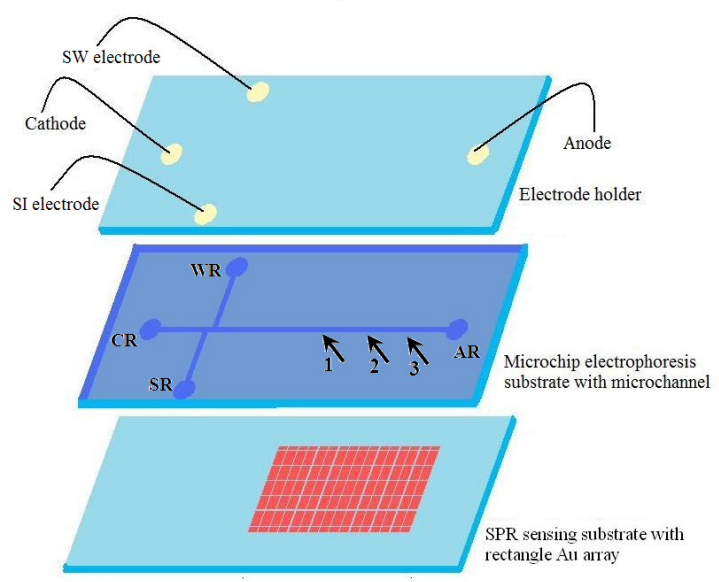

Fig. 1. Schematic of the integrated microchip with $S P R$-sensing gold microarray.

The microchip electrophoresis procedure was implemented on the integrated chip using a home-made high-voltage power supply. TBE buffer solution with $0.5 \%$ HPMC and 10\% methanol was filled all the microchannels and reservoirs, and a sample of FITC was added in the sample reservoir. In the sample loading procedure, $120 \mathrm{~V}$ was applied between the sample inlet and the sample waste (Fig. 1). Following sample injection over $50 \mathrm{~s}$, subsequent electrophoresis process was implemented under $100 \mathrm{~V} / \mathrm{cm}$ electric field strength between cathode and anode. The moving process of FITC driven by electrokinetic force was continuously detected by our SPRI instrument.
A home-made SPR imaging (SPRI) instrument, which was working with Kretschmann configuration to achieve the resonant condition by total internal reflection [10], was used for SPR imaging experiments. In the SPR imaging instrument, an intensity-stabilized, He-Ne laser with a wavelength of $650 \mathrm{~nm}$ was used as a light source, which was installed on a circumrotated arm. The laser beam was expanded and collimated by optical lens before traveling through a triangular prism and the SPR-sensing substrate. The reflected light was detected by a charge coupled device (CCD) detector, which was fixed on another circumrotated arm. Both circumrotated arms are controlled by the stepper motor and the coding system. The incident angle changed from 40 to 70 degree and the incident angle range satisfied the needs for most biochemical experiment. The SPR imaging instrument can continuously detect the change of the solution refractive index at the fixed incident angle. Intensity changes in regions of interest selected from the image were monitored as a function of time to generate real-time response curves.

\section{Results and Conclusion}

Before the integrated chips were implemented an electrophoretic process with our SPR image systems, the stability of the SPR sensing gold film was firstly tested. The whole process of the electrochemical corrosion of Au was observed by the microscope. The SPR sensing gold film immersed in the solution was suffered from the high electric field. During the experiments, it was observed that the electrochemical corrosion process of $\mathrm{Au}$ usually included two steps: producing bubbles by electrolysis, then dissolving $\mathrm{Au}$. The $\mathrm{Au}$ at the corners always was attacked at first. Also from the results of experiments, it was found that the SPR sensing gold film of a rectangle microarray was able of withstand $100 \mathrm{~V} / \mathrm{cm}$ electric field strength without failure, which was up to approximately 2 times that of the square gold segments in big size [9]. And the intact gold film couldn't be stable under more than $20 \mathrm{~V} / \mathrm{cm}$ electric field strength. The possible reason might be that the rectangle design in smaller size would inhibit the polarization of $\mathrm{Au}$ which would be the intrinsic force for electrochemical corrosion.

The SPR characteristic of $\mathrm{Au}$ rectangle microarray has been tested. The SPR-sensing substrates with gold rectangle microarray, which were directly mounted in the home-made SPR imaging instrument, were tested by using the angle-scanning attenuated total reflection method. After deionized water was dropped on the Au surface, the resonant absorption peak was measured and used to evaluate the SPR 
characteristic of our SPR-sensing substrates with different $\mathrm{Au}$ rectangle microarray, when the incident angle was changed from 59.8 to 64 degree. The experimental results were shown in Fig. 2. From these results, the locations of the resonant absorption peak of different gold rectangle microarray were similar.

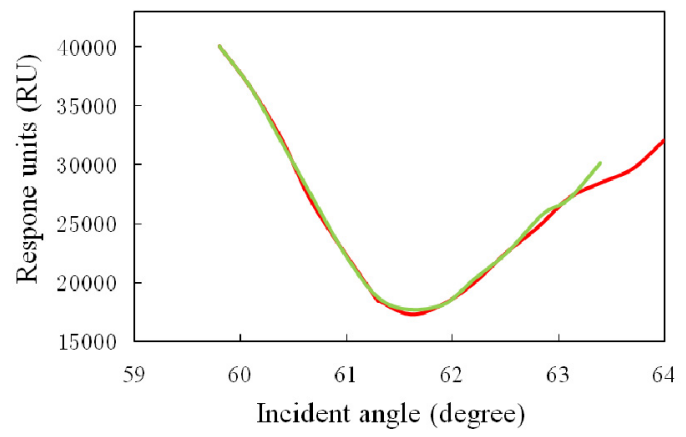

Fig. 2. The experiment results of SPR absorption peak with an intact gold film ("Green line"), a $150 \mu m \times 100 \mu m$ gold microarray ("Red line")

Finally, the integrated chip was mounted on our SPR image systems, and then the anglescanning attenuated total reflection method was used to get the resonant absorption peak and chose an optimal incident angle. After that the region at the end of the separation channel was selected as the detection point (show in Fig. 1), and the light intensity was continuously monitored. The moving process of FITC driven by electrokinetic force is continuously tested (Fig. 3), which proves the possibility of electrokinetic fluid transport with SPR imaging detection system with a relatively high electric field strength. With SPR image technology, the process of electrophoresis separation will be measured, which could be a label-free detection method for molecular analysis.

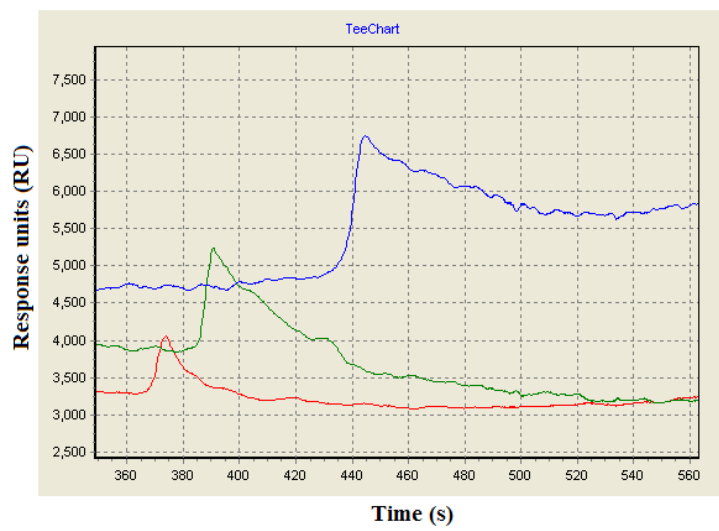

Fig. 3. The detection of FITC at different locations of the microchannel. "Red line": SPR curve at "1" of Fig. 1; "Green line": SPR curve at "2" of Fig. 1; "Blue line": SPR curve at "3" of Fig. 1.

\section{Acknowledgements}

The authors greatly acknowledge the financial support from the National Science Foundation of China under Grant number 31100820, 61176112 and 60701019. This work is also sponsored by the Major National Scientific Research Plan under Grant number 2011 CB933202.

\section{References}

[1] A.J. DeMello, Control and detection of chemical reactions in microfluidic systems, Nature 442, 394-402 (2006).

[2] H. Craighead, Future lab-on-a-chip technologies for interrogating individual molecules, Nature 442, 387-393(2006).

[3] A. Arora, Latest Developments in Micro Total Analysis, Anal Chem 82, 4830-4847(2010).

[4] P. Yager, T. Edwards, E. Fu, K. Helton, K. Nelson, M.R. Tam, B.H. Weigl, Microfluidic diagnostic technologies for global public health, Nature 442, 412-418(2006).

[5] G.M. Whitesides, The origins and the future of microfluidics, Nature 442, 368-373(2006).

[6] A. Abbas, M.J. Linman, Q. Cheng, New trends in instrumental design for surface plasmon resonance-based biosensors, Biosensors \& bioelectronics 26, 1815-1824 (2011).

[7] S. Scarano, C. Scuffi, M. Mascini, M. Minunni, Surface plasmon resonance imaging (SPRi)based sensing: a new approach in signal sampling and management, Biosensors \& bioelectronics $26,1380-1385(2010)$.

[8] C. C. Liu, D. F. Cui, H. Li, A hard-soft microfluidic-based biosensor flow cell for SPR imaging application Biosensors and Bioelectronics 26, 255-261 (2010)

[9] N. Ly, K. Foley, N. Tao, Integrated Label-Free Protein Detection and Separation in Real Time Using Confined Surface Plasmon Resonance Imaging, Analytical Chemistry 79, 2546-2551 (2007)

[10] D. Cui, Cai, H., Wang, J., Li, Y., Liang, J., Liu, C., Wang, Y., Zheng, Z., Chinese Patent Appl. No. CN 200610066542.4., (2006). 